\title{
Some variants of contraction principle in the case of operators with Volterra property: step by step contraction principle
}

Ioan A. Rus ${ }^{\mathrm{a}}$

${ }^{a}$ Department of Mathematics, Babeș-Bolyai University, 1, Kogălniceanu Street, 400084,

Cluj-Napoca, Romania.

\begin{abstract}
Following the idea of T.A. Burton, of progressive contractions, presented in some examples (T.A. Burton, $A$ note on existence and uniqueness for integral equations with sum of two operators: progressive contractions, Fixed Point Theory, 20 (2019), No. 1, 107-113) and the forward step method (I.A. Rus, Abstract models of step method which imply the convergence of successive approximations, Fixed Point Theory, 9 (2008), No. 1, 293-307), in this paper we give some variants of contraction principle in the case of operators with Volterra property. The basic ingredient in the theory of step by step contraction is G-contraction (I.A. Rus, Cyclic representations and fixed points, Ann. T. Popoviciu Seminar of Functional Eq. Approxim. Convexity, 3 (2005), 171-178). The relevance of step by step contraction principle is illustrated by applications in the theory of differential and integral equations.
\end{abstract}

Keywords: Space of continuous function, operator with Volterra property, max-norm, Bielecki norm, contraction, $G$-contraction, fiber contraction, progressive contraction, step by step contraction, fixed point, Picard operator, weakly Picard operator, differential equation, integral equation, conjecture. 2010 MSC: 47H10, 47H09, 34K05, 34K12, 45D05, 45G10, 54H25.

\section{Introduction}

Following an idea of T.A. Burton ([7], [8], [9], ...) of progressive contractions, and the forward step method $([21])$, in this paper we give some variants of contraction principle in the case of operators with

Email address: iarus@math.ubbcluj.ro (Ioan A. Rus ) 
Volterra property. The basic ingredient in our variant, step by step contraction principle, is $G$-contraction ([20]). Some applications to differential and integral equations are also given. In connection with our abstract results, a conjecture is formulated.

\section{Preliminaries}

\section{1. $G$-contractions}

Let $(X, d)$ be a metric space and $G \subset X \times X$ be a nonempty subset. An operator $f: X \rightarrow X$ is a $G$-contraction if there exists $l \in] 0,1[$ such that,

$$
d(f(x), f(y)) \leq l d(x, y), \forall(x, y) \in G .
$$

Here are some examples of subsets $G \subset X \times X$ :

(1) $G:=G(f)$, the graphic of the operator $f$. In this case, a $G$-contraction is a graphic contraction ([17], $[24], \ldots)$.

(2) Let $A_{i} \subset X, i=\overline{1, p}$, be nonempty closed subsets such that:

(i) $X=\bigcup_{i=1}^{p} A_{i}$;

(ii) $f\left(A_{i}\right) \subset A_{i+1}, i=\overline{1, p},\left(A_{p+1}=A_{1}\right)$.

For, $G:=\bigcup_{i=1}^{p}\left(A_{i} \times A_{i+1}\right)$, a $G$-contraction is a cyclic contraction of Kirk-Srinivasan-Veeramani (see the references in [20]).

(3) Let $a, b, c \in \mathbb{R}, a<c<b$ and $X:=C[a, b]$ with $d(x, y):=\max _{t \in[a, b]}|x(t)-y(t)|$. For $K, H \in C([a, b] \times$ $[a, b] \times \mathbb{R}, \mathbb{R})$, we consider the operator, $f: C[a, b] \rightarrow C[a, b]$, defined by,

$$
f(x)(t):=\int_{a}^{c} K(t, s, x(s)) d s+\int_{a}^{t} H(t, s, x(s)) d s, t \in[a, b] .
$$

We suppose that there exists $L_{H}>0$ such that

$$
|H(t, s, u)-H(t, s, v)| \leq L_{H}|u-v|, \forall t, s \in[a, b], \forall u, v \in \mathbb{R} .
$$

If, $L_{H}(b-c)<1$ and if we take

$$
G:=\left\{(x, y) \in C[a, b] \times C[a, b]|x|_{[a, c]}=\left.y\right|_{[a, c]}\right\},
$$

then $f$ is a $G$-contraction.

For other examples of $G$-contractions see [20] and [24], pp. 282-284.

\subsection{Weakly Picard operators}

Let $(X, \rightarrow)$ be an $L$-space $((X, d), \stackrel{d}{\rightarrow} ;(X, \tau), \stackrel{\tau}{\rightarrow} ;(X,\|\cdot\|), \stackrel{\|\cdot\|}{\rightarrow}, \rightarrow ; \ldots)$. An operator $f: X \rightarrow X$ is weakly Picard operator $(W P O)$ if the sequence, $\left(f^{n}(x)\right)_{n \in \mathbb{N}}$, converges for all $x \in X$ and the limit (which generally depend on $x$ ) is a fixed point of $f$. $(P O)$.

If an operator $f$ is $W P O$ and the fixed point set of $f, F_{f}=\left\{x^{*}\right\}$, then by definition $f$ is Picard operator

For a $W P O, f: X \rightarrow X$, we define the operator $f^{\infty}: X \rightarrow X$, by $f^{\infty}(x):=\lim _{n \rightarrow \infty} f^{n}(x)$.

We remark that, $f^{\infty}(X)=F_{f}$, i.e., $f^{\infty}$ is a set retraction of $X$ on $F_{f}$.

For the case of ordered $L$-spaces, we have some properties of $W P O$ and $P O$.

Abstract Gronwall Lemma. Let $(X, \rightarrow, \leq)$ be an ordered $L$-space and $f: X \rightarrow X$ be an operator. We suppose that: 
(1) $f$ is increasing;

(2) $f$ is $W P O$.

Then:

(i) $x \leq f(x) \Rightarrow x \leq f^{\infty}(x)$;

(ii) $x \geq f(x) \Rightarrow x \geq f^{\infty}(x)$.

Abstract Comparison Lemma. Let $(X, \rightarrow, \leq)$ be an ordered $L$-space and $f, g, h: X \rightarrow X$ be such that:

(1) $f \leq g \leq h$;

(2) the operators $f, g, h$ are $W P O$;

(3) the operator $g$ is increasing.

Then:

$$
x \leq y \leq z \Rightarrow f^{\infty}(x) \leq g^{\infty}(y) \leq h^{\infty}(z) .
$$

Regarding the theory of $W P O$ and $P O$ see [18], [19], [22], [23], [26], [17], [24], [2], ..

\subsection{Fiber Contraction Principle}

In order to present our results, we need the following theorems (see [22], [25], [26], [27], ...).

Fiber Contraction Theorem. Let $(X, \rightarrow)$ be an $L$-space, $(Y, \rho)$ be a metric space, $g: X \rightarrow X, h:$ $X \times Y \rightarrow Y$ and $f: X \times Y \rightarrow X \times Y, f(x, y):=(g(x), h(x, y))$. We suppose that:

(1) $(Y, \rho)$ is a complete metric space;

(2) $g$ is $W P O$;

(3) $h(x, \cdot): Y \rightarrow Y$ is $l$-contraction, $\forall x \in X$;

(4) $h: X \times Y \rightarrow Y$ is continuous.

Then, $f$ is WPO. Moreover, if $g$ is a $P O$, then $f$ is a $P O$.

Generalized Fiber Contraction Theorem. Let $(X, \rightarrow)$ be an $L$-space, $\left(X_{i}, d_{i}\right), i=\overline{1, m}, m \geq 1$ be metric spaces. Let, $f_{i}: X_{0} \times \ldots \times X_{i} \rightarrow X_{i}, i=\overline{0, m}$, be some operators. We suppose that:

(1) $\left(X_{i}, d_{i}\right), i=\overline{1, m}$, are complete metric spaces;

(2) $f_{0}$ is a $W P O$;

(3) $f_{i}\left(x_{0}, \ldots, x_{i-1}, \cdot\right): X_{i} \rightarrow X_{i}, i=\overline{1, m}$, are $l_{i}$-contractions;

(4) $f_{i}, i=\overline{1, m}$, are continuous.

Then, the operator $f: X_{0} \times \ldots \times X_{m} \rightarrow X_{0} \times \ldots \times X_{m}$, defined by,

$$
f\left(x_{0}, \ldots, x_{m}\right):=\left(f_{0}\left(x_{0}\right), f_{1}\left(x_{0}, x_{1}\right), \ldots, f_{m}\left(x_{0}, \ldots, x_{m}\right)\right)
$$

is a $W P O$.

If $f_{0}$ is a $P O$, then $f$ is a $P O$. 


\section{Operators with Volterra property with respect to a subinterval}

Let $(\mathbb{B},+, \mathbb{R},|\cdot|)$ be a Banach space, $a, b, c \in \mathbb{R}, a<c<b$. In what follows, we consider on $C([a, b], \mathbb{B})$, $C([a, c], \mathbb{B})$ norms of uniform convergence (max-norm, $\|\cdot\|$, Bielecki norm, $\left.\|\cdot\|_{\tau}\right)$. In, $C([a, b], \mathbb{B}) \times C([a, b], \mathbb{B})$, we consider a subset defined by,

$$
G:=\left\{(x, y)|x, y \in C([a, b], \mathbb{B}), x|_{[a, c]}=\left.y\right|_{[a, c]}\right\},
$$

and in, $C([a, b], \mathbb{B})$, for each $x \in C([a, c], \mathbb{B})$ we consider the subset,

$$
X_{x}:=\left\{y \in C([a, b], \mathbb{B})|y|_{[a, c]}=x\right\} .
$$

Definition 3.1. An operator, $V: C([a, b], \mathbb{B}) \rightarrow C([a, b], \mathbb{B})$, has the Volterra property with respect to the subinterval, $[a, c]$, if the following implication holds,

$$
x, y \in C([a, b], \mathbb{B}),\left.x\right|_{[a, c]}=\left.\left.y\right|_{[a, c]} \Rightarrow V(x)\right|_{[a, c]}=\left.V(y)\right|_{[a, c]} .
$$

Definition 3.2. An operator, $V: C([a, b], \mathbb{B}) \rightarrow C([a, b], \mathbb{B})$, has the Volterra property if it has the Volterra property with respect to each subinterval, $[a, t]$, for $a<t<b$.

For example, let $K, H \in C([a, b] \times[a, b] \times \mathbb{B}, \mathbb{B})$ and $V: C([a, b], \mathbb{B}) \rightarrow C([a, b], \mathbb{B})$ be defined by,

$$
V(x)(t):=\int_{a}^{c} K(t, s, x(s)) d s+\int_{a}^{t} H(t, s, x(s)) d s, t \in[a, b] .
$$

This operator has the Volterra property with respect to the subinterval $[a, c]$, but $V$ has not the Volterra property.

If, $V: C([a, b], \mathbb{B}) \rightarrow C([a, b], \mathbb{B})$, is an operator with Volterra property with respect to $[a, c]$, then the operator $V$ induces an operator, $V_{1}$, on $C([a, c], \mathbb{B})$, defined by

$$
V_{1}(x):=\left.V(\tilde{x})\right|_{[a, c]} \text {, where } \tilde{x} \in C([a, b], \mathbb{B}) \text { with, }\left.\tilde{x}\right|_{[a, c]}=x \text {. }
$$

Remark 3.3. If $V$ has the Volterra property with respect to $[a, c]$ and $V$ is a $G$-contraction (see section 2.1.), then the operator

$$
\left.V\right|_{X_{x}}: X_{x} \rightarrow X_{V_{1}(x)}
$$

is a contraction for all $x \in C([a, c], \mathbb{B})$. If $x^{*} \in F_{V_{1}}$, then, $V\left(X_{x^{*}}\right) \subset X_{x^{*}}$.

The first abstract result of our paper is the following.

Theorem 3.4. In terms of the above notations, we suppose that:

(1) $V$ has the Volterra property with respect to $[a, c]$;

(2) $V_{1}$ is a contraction;

(3) $V$ is a G-contraction.

Then:

(i) $F_{V}=\left\{x^{*}\right\}$;

(ii) $\left.x^{*}\right|_{[a, c]}=V_{1}^{\infty}(x), \forall x \in C([a, c], \mathbb{B})$;

(iii) $x^{*}=V^{\infty}(x), \forall x \in X_{\left.x^{*}\right|_{[a, c]}}$. 
Proof. From (1) we have that, $F_{V_{1}}=\left\{x_{1}^{*}\right\}, x_{1}^{*} \in C([a, c], \mathbb{B})$. From (3) and Remark $3.3,\left.V\right|_{X_{x_{1}^{*}}}: X_{x_{1}^{*}} \rightarrow X_{x_{1}^{*}}$, is a contraction, i.e., it has a unique fixed point, $x^{*}$, and $\left.x^{*}\right|_{[a, c]}=x_{1}^{*}$. From these we have $(i),(i i)$ and (iii).

Conjecture 3.5. In the conditions of Theorem 3.4, the operator $V$ is $P O$, i.e., $x^{*}=V^{\infty}(x), \forall x \in$ $C([a, b], \mathbb{B})$.

For a better understanding of Theorem 3.4 and Conjecture 3.5, in what follows, we present some examples.

Example 3.6. Let $a, b, c$ be as above and $\mathbb{B}:=\mathbb{R}$. For $K, H \in C([a, b] \times[a, b] \times \mathbb{R}, \mathbb{R})$ we consider the following functional integral equation,

$$
x(t)=\int_{a}^{c} K(t, s, x(s)) d s+\int_{a}^{t} H\left(t, s, \max _{\theta \in[a, s]} x(\theta)\right) d s, t \in[a, b] .
$$

We are looking for the solution of this equation in $C[a, b]$. In addition, we suppose that:

$\left(2^{\prime}\right)$ there exists $L_{K}>0$ such that:

$$
|K(t, s, u)-K(t, s, v)| \leq L_{K}|u-v|, \forall t \in[a, b], \forall s \in[a, c], \forall u, v \in \mathbb{R}
$$

$\left(3^{\prime}\right)$ there exists $L_{H}>0$ such that,

$$
|H(t, s, u)-H(t, s, v)| \leq L_{H}|u-v|, \forall t, s \in[a, b], \forall u, v \in \mathbb{R} .
$$

In this case:

$$
\begin{aligned}
V(x)(t) & =\text { the second part of } 3.1 ; \\
V_{1}(x)(t) & =\text { the second part of } 3.1, \text { for } t \in[a, c] .
\end{aligned}
$$

It is clear that $V$ has the Volterra property with respect to the subinterval $[a, c]$.

We consider on $C[a, c]$ and $C[a, b]$ max-norms and if, $\left(L_{K}+L_{H}\right)(c-a)<1$, the operator $V_{1}$ is a contraction and if, $L_{H}(b-c)<1$, the operator $V$ is a $G$-contraction.

So, by Theorem 3.4, in the above conditions, equation (3.1) has in $C[a, b]$ a unique solution, $x^{*}$. Moreover, for $t \in[a, c], x^{*}(t)=\lim _{n \rightarrow \infty} x_{n}(t)$, for each $x_{0} \in C[a, c]$, where $\left\{x_{n}\right\}_{n \in \mathbb{N}}$ is defined by,

$$
x_{n+1}(t)=\int_{a}^{c} K\left(t, s, x_{n}(s)\right) d s+\int_{a}^{t} H\left(t, s, \max _{\theta \in[a, s]} x_{n}(\theta)\right) d s,
$$

and for $t \in[a, b], x^{*}(t)=\lim _{n \rightarrow \infty} y_{n}(t)$, where $\left\{y_{n}\right\}_{n \in \mathbb{N}}$, is defined by

$y_{0} \in C[a, b]$, with $\left.y_{0}\right|_{[a, c]}=\left.x^{*}\right|_{[a, c]}$, and

$$
y_{n+1}(t)=\int_{a}^{c} K\left(t, s, x^{*}(s)\right) d s+\int_{a}^{t} H\left(t, s, \max _{\theta \in[a, s]} y_{n}(\theta)\right) d s .
$$

Remark 3.7. In the case of operator $V$, in this example, Conjecture 3.5 is a theorem. Indeed, let $X_{0}:=$ $C[a, c], X_{1}:=C[c, b]$ and $C[a, b]$ be endowed with max-norms. We take, $f_{0}:=V_{1}$ and $f_{1}(x, y): C[a, c] \times$ $C[c, b] \rightarrow C[c, b]$ be defined by

$$
\begin{aligned}
f_{1}(x, y)(t) & :=\int_{a}^{c} K(t, s, x(s)) d s+\int_{a}^{c} H\left(t, s, \max _{\theta \in[a, s]} x(\theta)\right) d s+ \\
& +\int_{c}^{t} H\left(t, s, \max \left(\max _{\theta \in[a, c]} x(\theta), \max _{\theta \in[c, s]} y(\theta)\right)\right) d s .
\end{aligned}
$$


We remark that, $f_{0}$ is a $P O$, and $f_{1}(x, \cdot): C[c, b] \rightarrow C[c, b]$ is $L_{H}(b-c)$-contraction. By Fiber Contraction Theorem, in the conditions, $\left(L_{K}+L_{H}\right)(c-a)<1$ and $L_{H}(b-c)<1$, the operator $f$ is a Picard operator. Let,

$$
x_{0} \in C[a, c], x_{n+1}=f_{0}\left(x_{n}\right), n \in \mathbb{N},
$$

and

$$
y_{0} \in C[c, b], y_{n+1}=f_{1}\left(x_{n}, y_{n}\right), n \in \mathbb{N} .
$$

Then, $\left.x_{n} \rightarrow x^{*}\right|_{[a, c]}$ as $n \rightarrow \infty,\left.y_{n} \rightarrow x^{*}\right|_{[c, b]}$ as $n \rightarrow \infty$.

We denote,

$$
u_{n}(t)=\left\{\begin{array}{l}
x_{n}(t), t \in[a, c], \\
y_{n}(t), t \in[c, b] .
\end{array}\right.
$$

Then, $u_{n} \in C[a, b]$, for $n \in \mathbb{N}^{*}$, and, $u_{n+1}=V\left(u_{n}\right)$ with $u_{n} \rightarrow x^{*}$ as $n \rightarrow \infty$, i.e., $V$ is a PO.

This result is very important because we can apply for $V$, the Abstract Gronwall Lemma. So we have:

Theorem 3.8. Let us consider the equation (3.1) in the following conditions: $\left(L_{K}+L_{H}\right)(c-a)<1$, $L_{H}(b-c)<1$ and $K(t, s, \cdot), H(t, s, \cdot): \mathbb{R} \rightarrow \mathbb{R}$ are increasing functions, for all $t, s \in[a, b]$. Let us denote by $x^{*}$ the unique solution of 3.1. Then the following implications hold:

(i) $x \in C[a, b], x(t) \leq \int_{a}^{c} K(t, s, x(s)) d s+\int_{a}^{t} H\left(t, s, \max _{\theta \in[a, s]} x(\theta)\right) d s, t \in[a, b], \Rightarrow x \leq x^{*} ;$

(ii) $x \in C[a, b], x(t) \geq \int_{a}^{c} K(t, s, x(s)) d s+\int_{a}^{t} H\left(t, s, \max _{\theta \in[a, s]} x(\theta)\right) d s, t \in[a, b], \Rightarrow x \geq x^{*}$.

Also, from the Abstract Comparison Lemma we have a comparison result for equation (3.1).

Remark 3.9. For the functional integral equations with maxima, see [1], [11], [16], [22], [13], ...

Example 3.10. Let $a, b, c \in \mathbb{R}, a<b<c$, and $(\mathbb{B},+, \mathbb{R},|\cdot|)$ be a Banach space. For $K, H \in C([a, b] \times[a, b] \times$ $\mathbb{B}, \mathbb{B})$ we consider the following integral equation,

$$
x(t)=\int_{a}^{c} K(t, s, x(s)) d s+\int_{a}^{t} H(t, s, x(s)) d s, t \in[a, b] .
$$

We are looking for solutions of these equations in $C([a, b], \mathbb{B})$. To do this, in addition, we suppose that:

$\left(2^{\prime \prime}\right)$ there exists $L_{K}>0$ such that,

$$
|K(t, s, u)-K(t, s, v)| \leq L_{K}|u-v|, \forall t \in[a, b], \forall s \in[a, c], \forall u, v \in \mathbb{B} ;
$$

$\left(3^{\prime \prime}\right)$ there exists $L_{H}>0$ such that,

$$
|H(t, s, u)-H(t, s, v)| \leq L_{H}|u-v|, \forall t, s \in[a, b], \forall u, v \in \mathbb{B} .
$$

In the case of equation (3.2) we have:

$$
\begin{aligned}
V(x)(t) & =\text { the second part of } 3.2 ; \\
V_{1}(x)(t) & =\text { the second part of } 3.2
\end{aligned}
$$

First, we remark that $V$ has the Volterra property with respect to the subinterval $[a, c]$.

If we consider on $(C[a, c], \mathbb{B})$ and $C[a, b]$ max-norms, then if, $\left(L_{K}+L_{H}\right)(c-a)<1$, the operator $V_{1}$ is a contraction (i.e., $\left.P O\right)$ and if, $L_{H}(b-c)<1$, the operator $V$ is a G-contraction. By Theorem 
3.4. in these conditions, equation $(3.2)$ has in $C([a, b], \mathbb{B})$ a unique solution, $x^{*}$. Moreover, for $t \in[a, c]$, $x^{*}(t)=\lim _{n \rightarrow \infty} x_{n}(t)$, where $x_{0} \in C[a, c]$,

$$
x_{n+1}(t)=\int_{a}^{c} K\left(t, s, x_{n}(s)\right) d s+\int_{a}^{t} H\left(t, s, x_{n}(s)\right) d s, n \in \mathbb{N}
$$

and for $t \in[a, b], x^{*}(t)=\lim _{n \rightarrow \infty} y_{n}(t)$, where $y_{0} \in C([a, b], \mathbb{B})$, with $\left.y_{0}\right|_{[a, c]}=x^{*}$, and

$$
y_{n+1}(t)=\int_{a}^{c} K\left(t, s, x^{*}(s)\right) d s+\int_{a}^{t} H\left(t, s, y_{n}(s)\right) d s, n \in \mathbb{N} .
$$

Remark 3.11. In a similar way, as in the case of Example 3.6, the Conjecture 3.5 is a theorem for the operator $V$ in Example 3.10 .

Remark 3.12. We can work, in the case of Example 3.10 with max-norm on $C([a, c], \mathbb{B})$ and with a Bielecki norm on $C[c, b]$, i.e., on $C([a, b], \mathbb{B})$ with the norm, $\|x\|=\max \left(\max _{t \in[a, c]}|x(t)|, \max _{t \in[c, b]} e^{-\tau(t-c)}|x(t)|\right)$.

If $\mathbb{B}:=\mathbb{R}^{m}$, then we can work with vectorial max-norms and with vectorial Bielecki norms.

Remark 3.13. For example of integral operator like $V$ in Example 3.10, which appear in differential equations, see: [5], [14], [4], [3] and the references in [3].

\section{Operators with Volterra property}

Let, $V: C([a, b], \mathbb{B}) \rightarrow C([a, b], \mathbb{B})$, be an operator with Volterra property. Let $m \in \mathbb{N}, m \geq 2, t_{0}:=a$, $t_{1}:=t_{0}+\frac{b-a}{m}, \ldots, t_{k}:=t_{0}+\frac{k(b-a)}{m}, \ldots, t_{m}:=b$. We denote by $V_{k}: C\left(\left[t_{0}, t_{k}\right], \mathbb{B}\right) \rightarrow C\left(\left[t_{0}, t_{k}\right], \mathbb{B}\right)$, $k=\overline{1, m-1}$, the operators induced by $V$ on $\left[t_{0}, t_{k}\right]$ (see the definition of $V_{1}$ in section 3 ). We also consider the following sets,

$$
G_{k}:=\left\{(x, y)\left|x, y \in C\left(\left[t_{0}, t_{k+1}\right], \mathbb{B}\right), x\right|_{\left[t_{0}, t_{k}\right]}=\left.y\right|_{\left[t_{0}, t_{k}\right]}\right\}, k=\overline{1, m-1} .
$$

For, $x_{k} \in C\left(\left[t_{0}, t_{k}\right], \mathbb{B}\right), k=\overline{1, m-1}$, we denote,

$$
X_{x_{k}}:=\left\{y \in C\left(\left[t_{0}, t_{k+1}\right], \mathbb{B}\right)|y|_{\left[t_{0}, t_{k}\right]}=x_{k}\right\} .
$$

The second basic result of this paper is the following.

Theorem 4.1 (Theorem of step by step contraction). We suppose that:

(1) V has the Volterra property;

(2) $V_{1}$ is a contraction;

(3) $V_{k}$ is a $G_{k-1}$-contraction, for $k=\overline{2, m}$.

Then:

(i) $F_{V}=\left\{x^{*}\right\}$;

(ii)

$$
\begin{aligned}
\left.x^{*}\right|_{\left[t_{0}, t_{1}\right]} & =V_{1}^{\infty}(x), \quad \forall x \in C\left(\left[t_{0}, t_{1}\right], \mathbb{B}\right), \\
\left.x^{*}\right|_{\left[t_{0}, t_{2}\right]} & =V_{2}^{\infty}(x), \quad \forall x \in X_{\left.x^{*}\right|_{\left[t_{0}, t_{1}\right]},}, \\
& \vdots \\
\left.x^{*}\right|_{\left[t_{0}, t_{m-1}\right]} & =V_{m-1}^{\infty}(x), \quad \forall x \in X_{\left.x^{*}\right|_{\left[t_{0}, t_{m-2}\right]}} .
\end{aligned}
$$


(iii) $x^{*}=V^{\infty}(x),\left.\forall x \in X_{x^{*}}\right|_{\left[t_{0}, t_{m-1}\right]}$.

Proof. It follows from successive (step by step !) application of Theorem 3.4, for the pairs, $\left(V_{k+1}, V_{k}\right)$, $k=\overline{1, m-1}$, with $V_{k+1}$ as $V$ and $V_{k}$ as $V_{1}$.

Conjecture 4.2. In the condition of Theorem 4.1 the operator $V$ is PO, with respect to uniform convergence on $C([a, b], \mathbb{B})$.

Example 4.3. For $K \in C([a, b] \times[a, b] \times \mathbb{R})$ we consider the following functional integral equation with maxima,

$$
x(t)=\int_{a}^{t} K\left(t, s, \max _{\theta \in[a, s]} x(\theta)\right) d s, t \in[a, b]
$$

By step by step contraction principle we shall prove that, if there exists $L_{K}>0$ such that,

$$
|K(t, s, u)-K(t, s, v)| \leq L_{K}|u-v|, \forall t, s \in[a, b], \forall u, v \in \mathbb{R},
$$

then the equation (4.1) has in $C[a, b]$ a unique solution.

Indeed, let $m \in \mathbb{N}^{*}$ be such that, $\frac{L_{K}(b-a)}{m}<1$. Let, $V: C[a, b] \rightarrow C[a, b]$ be defined by,

$$
V(x)(t):=\text { the second part of 4.1. }
$$

First, we remark that $V$ has the Volterra property. In this case:

$$
V_{1}: C\left[t_{0}, t_{1}\right] \rightarrow C\left[t_{0}, t_{1}\right], V_{1}(x)(t)=\int_{t_{0}}^{t_{1}} K\left(t, s, \max _{\theta \in\left[t_{0}, s\right]} x(\theta)\right) d s, t \in\left[t_{0}, t_{1}\right] .
$$

A Lipschitz constant for $V_{1}$ is, $\frac{L_{K}(b-a)}{m}$. So, $V_{1}$ is a contraction with respect to max-norm.

In a similar way, $V_{2}$ is a $G_{1}$-contraction, $V_{k}$ is a $G_{k-1}$-contraction and $V$ is $G_{m-1}$-contraction.

So, we are in the conditions of Theorem 4.1. From this theorem we have that:

The equation (4.1) has in $C[a, b]$ a unique solution, $x^{*}$. Moreover,

- for $t \in\left[t_{0}, t_{1}\right], x^{*}(t)=\lim _{n \rightarrow \infty} x_{n}(t)$, where $x_{0} \in C\left[t_{0}, t_{1}\right], x_{n+1}(t)=\int_{t_{0}}^{t} K\left(t, s, \max _{\theta \in\left[t_{0}, s\right]} x_{n}(\theta)\right) d s$;

- for $t \in\left[t_{0}, t_{2}\right], x^{*}(t)=\lim _{n \rightarrow \infty} x_{n}(t)$, where $x_{0} \in C\left[t_{0}, t_{2}\right]$ with $\left.x_{0}\right|_{\left[t_{0}, t_{1}\right]}=\left.x^{*}\right|_{\left[t_{0}, t_{1}\right]}$, and $x_{n+1}(t)=$ $\int_{t_{0}}^{t} K\left(t, s, \max _{\theta \in\left[t_{0}, s\right]} x_{n}(\theta)\right) d s, n \in \mathbb{N}$;

- for $t \in\left[t_{0}, t_{m}\right], x^{*}(t)=\lim _{n \rightarrow \infty} x_{n}(t)$, where $x_{0} \in C\left[t_{0}, t_{m}\right]$ with $\left.x_{0}\right|_{\left[t_{0}, t_{m-1}\right]}=\left.x^{*}\right|_{\left[t_{0}, t_{m-1}\right]}$, and $x_{n+1}(t)=$ $\int_{t_{0}}^{t} K\left(t, s, \max _{\theta \in\left[t_{0}, s\right]} x_{n}(\theta)\right) d s$.

Remark 4.4. In a similar way as in the Example 3.6, by Generalized fiber contraction theorem, we have that, for $V$ in Example 4.3, the Conjecture 4.2 is a theorem.

Example 4.5. For $f \in C([a, b] \times \mathbb{R})$, we consider the following Cauchy problem

$$
\begin{aligned}
& x^{\prime}(t)=f\left(t, \max _{\theta \in[a, t]} x(\theta)\right), t \in[a, b] \\
& x(a)=0
\end{aligned}
$$

This problem with $x \in C^{1}[a, b]$ is equivalent with the following functional integral equation with maxima, in $C[a, b]$,

$$
x(t)=\int_{a}^{t} f\left(s, \max _{\theta \in[a, s]} x(\theta)\right) d s,
$$


From the result, in Example 4.3, we have that, if there exists $L_{f}>0$ such that,

$$
|f(t, u)-f(t, v)| \leq L_{f}|u-v|, \forall t \in[a, b], \forall u, v \in \mathbb{R},
$$

then the equation (4.3) has in $C[a, b]$ a unique solution, i.e., the Cauchy problem (4.2) has in $C^{1}[a, b] a$ unique solution.

Remark 4.6. For functional differential equations see: [1], [6], [11], [12], [16], [22], ...

Remark 4.7. For operators with Volterra property see: [10], [21], [15] and the references therein.

\section{Step by step generalized contraction principles}

There is a large class of generalized contraction principle (see, for example, [24, [2], [17]). As an example in what follows, we consider the case of $\varphi$-contractions.

Let $(X, d)$ be a metric space, $G \subset X \times X$ a nonempty subset and $f: X \rightarrow X$ be an operator.

Definition 5.1. Let $\varphi: \mathbb{R}_{+} \rightarrow \mathbb{R}_{+}$be a comparison function. By definition, $f$ is a $(G, \varphi)$-contraction if,

$$
d(f(x), f(y)) \leq \varphi(d(x, y)), \forall x, y \in G .
$$

In the terms of notations in section 4 , in a similar way as in the case of Theorem 4.1, we have:

Theorem 5.2 (Theorem of step by step $\varphi$-contraction). We suppose that:

(1) $V$ has the Volterra property;

(2) $V_{1}$ is a $\varphi$-contraction;

(3) $V_{k}$ is a $\left(G_{k-1}, \varphi\right)$-contraction, for $k=\overline{2, m}$.

Then:

(i) $F_{V}=\left\{x^{*}\right\}$;

(ii)

$$
\begin{aligned}
\left.x^{*}\right|_{\left[t_{0}, t_{1}\right]} & =V_{1}^{\infty}(x), \quad \forall x \in C\left(\left[t_{0}, t_{1}\right], \mathbb{B}\right), \\
\left.x^{*}\right|_{\left[t_{0}, t_{2}\right]} & =V_{2}^{\infty}(x), \quad \forall x \in X_{\left.x^{*}\right|_{\left[t_{0}, t_{1}\right]},} \\
& \vdots \\
\left.x^{*}\right|_{\left[t_{0}, t_{m-1}\right]} & =V_{m-1}^{\infty}(x), \quad \forall x \in X_{\left.x^{*}\right|_{\left[t_{0}, t_{m-2}\right]}} .
\end{aligned}
$$

(iii) $x^{*}=V^{\infty}(x),\left.\forall x \in X_{x^{*}}\right|_{\left[t_{0}, t_{m-1}\right]}$.

Problem 5.3. For which generalized contractions we have step by step corresponding result ? If such generalized contractions are found, then the problem is to give relevant applications of such result. 


\section{References}

[1] D.D. Bainov, S.G. Hristova, Differential Equations with Maxima, CRC Press, 2011.

[2] V. Berinde, Iterative Approximation of Fixed Points, Springer, 2007.

[3] O.-M. Bolojan, Fixed Point Methods for Nonlinear Differential Systems with Nonlocal Conditions, Casa Cărţii de Ştiinţă, Cluj-Napoca, 2013.

[4] A. Boucherif, First-order differential inclusions with nonlocal initial conditions, Appl. Math. Letters, 15 (2002), $409-414$.

[5] A. Boucherif, R. Precup, On the nonlocal initial value problem for first order differential equations, Fixed Point Theory, 4 (2003), 205-212.

[6] T.A. Burton, Stability by Fixed Point Theory for Functional Differential Equations, Dover Publ., New York, 2008.

[7] T.A. Burton, A note on existence and uniqueness for integral equations with sum of two operators: progressive contractions, Fixed Point Theory, 20 (2019), No. 1, 107-113.

[8] T.A. Burton, I.K. Purnaras, The shrinking fixed point map, Caputo and integral equations: progressive contraction, J. Fractional Calculus Appl., 9 (2018), No. 1, 188-194.

[9] T.A. Burton, I.K. Purnaras, Progressive contractions, product contractions, quadratic integro-differential equations, AIMS Math., 4 (2019), No. 3, 482-496.

[10] C. Corduneanu, Abstract Volterra equations: a survey, Mathematical and Computer Modeling, 32 (2000), 1503-1528.

[11] A. Halanay, Differential Equations: Stability, Oscillations, Time Lags, Acad. Press, New York, 1966.

[12] V. Kolmanovskii, A. Myshkis, Applied theory of functional-differential equations, Kluwer, 1992.

[13] V. Lakshmikantham, L. Wen, B. Zhang, Theory of Differential Equations with Unbounded Delay, Kluwer, 1994.

[14] O. Nica, R.Precup, On the nonlocal initial value problem for first order differential systems, Studia Univ. Babeş-Bolyai Math., 56 (2011), No. 3, 125-137.

[15] D. Otrocol, Ulam stabilities of differential equation with abstract Volterra operator in a Banach space, Nonlinear Funct. Anal. Appl., 15 (2010), No. 4, 613-619.

[16] D. Otrocol, I.A. Rus, Functional-differential equations with "maxima" via weakly Picard operator theory, Bull. Math. Soc. Sci. Math. Roumanie, 51 (2008), No. 3, 253-261.

[17] I.A. Rus, Generalized Contractions and Applications, Cluj Univ. Press, Cluj-Napoca, 2001.

[18] I.A. Rus, Picard operators and applications, Sc. Math. Jpn., 58 (2003), no. 1, 191-219.

[19] I.A. Rus, Fixed points, upper and lower fixed points: abstract Gronwall lemmas, Carpathian J. Math., 20 (2004), No. 1, 125-134.

[20] I.A. Rus, Cyclic reprezentations and fixed points, Ann. T. Popoviciu Seminar of Functional Eq. Approximation and Convexity, 3 (2005), 171-178.

[21] I.A. Rus, Abstract models of step method which imply the convergence of successive approximations, Fixed Point Theory, 9 (2008), No. 1, 293-307.

[22] I.A. Rus, Some nonlinear functional differential and integral equations, via weakly Picard operator theory: a survey, Carpathian J. Math., 26 (2010), No. 2, 230-258.

[23] I.A. Rus, Some problems in the fixed point theory, Adv. Theory of Nonlinear Analysis Appl., 2 (2018), No. 1, 1-10.

[24] I.A. Rus, A. Petruşel, G. Petruşel, Fixed Point Theory, Cluj Univ. Press, Cluj-Napoca, 2008.

[25] I.A. Rus, M.A. Şerban, Operators on infinite dimensional cartesian product, Analele Univ. de Vest Timişoara, 48 (2010), 253-263.

[26] I.A. Rus, M.A. Şerban, Basic problems of the metric fixed point theory and the relevance of a metric fixed point theorem, Carpathian J. Math., 29 (2013), No. 2, 239-258.

[27] M.A. Şerban, Teoria punctului fix pentru operatori definiţi pe produs cartezian, Presa Univ. Clujeană, Cluj-Napoca, 2002. 